\title{
Extreme increases in sleep duration raise T2DM risk
}

Women whose sleep duration increases by $\geq 2 \mathrm{~h}$ per day over a 14 -year period are more likely to develop type 2 diabetes mellitus (T2DM) than women whose sleep duration decreases or stays the same, according to new findings published in Diabetologia. Extreme changes in sleep length remained associated with T2DM risk despite extensive adjustment for lifestyle factors.

"Epidemiological studies show links between extremely long or short sleep duration and lower diet quality, lower physical activity and risk of obesity and T2DM," explains corresponding author Elizabeth Cespedes. However, whether the associations between sleep length and T2DM risk could be explained by lifestyle factors or weight gain, or whether sleep duration affected metabolic health via other mechanisms was unknown. The researchers also decided to investigate if changes in sleep duration modified T2DM risk, as previous studies had examined only single time points or failed to adequately adjust risk estimates. The researchers analysed data collected from 59,031 female nurses between 1986 and 2000 as part of the Nurses' Health Study. The self-reported data included sleep length, age, BMI, diet, illness and physical activity, and presence of T2DM was assessed in follow-up after 2000.

Cespedes and colleagues found that changes in sleep duration had modest, adverse associations with diet quality, weight gain and level of physical activity. However, even after adjustment for these factors and baseline sleep duration, women reporting increases of sleep duration $\geq 2 \mathrm{~h}$ per day between 1986 and 2000 had a higher risk of developing T2DM than women who reported no change (HR 1.15, 95\% CI 1.01-1.30). Women consistently sleeping $\leq 6 \mathrm{~h}$ per day were also at increased risk of T2DM compared with women consistently sleeping 7-8 h per day; however, this risk was attenuated after adjusting for BMI.

"Our results support the message that long-term maintenance of healthy sleep duration aids chronic disease prevention. However, increasing sleep duration after years of short sleep may not be a panacea," cautions Cespedes.

Hugh Thomas

ORIGINAL ARTICLE Cespedes, E. M. et al.

Long-term changes in sleep duration, energy balance and risk of type 2 diabetes. Diabetologia http://dx.doi.org/10.1007/s00125-015-3775-5 